\title{
Évolution saisonnière du potentiel hydrique et de la croissance de jeunes plants de Quercus rubra et de Quercus palustris au cours d'une sécheresse édaphique
}

\author{
J Timbal, C Lefebvre \\ INRA, laboratoire d'écophysiologie et nutrition, domaine de l'Hermitage, \\ Pierroton, 33610 Cestas, France
}

(Reçu le 24 mars 1993; accepté le 17 mars 1994)

\begin{abstract}
Résumé - Le chêne rouge (Quercus rubra $L$ ) est largement utilisé en France pour le reboisement, et son écophysiologie a déjà fait l'objet de nombreuse études, tant aux États-Unis qu'en France. Le chêne des marais (Quercus palustris Muench) semble cependant pouvoir avantageusement le remplacer dans certaines conditions si on en croit la réussite de certaines de ses introductions, en particulier dans le Sud-Ouest de la France. Malheureusement, ses caractéristiques écologiques et écophysiologiques sont bien moins connues. Nous avons donc comparé sa résistance à la sécheresse à celle du Chêne rouge lors d'une expérimentation sur semis, en conteneurs, par arrêt de l'irrigation soit après le premier cycle de croissance, soit après le second. Les mesures ont porté sur les surfaces foliaires développées, sur la croissance (en hauteur, en diamètre, en biomasse) et sur l'évolution saisonnière du potentiel hydrique. En condition d'alimentation hydrique non limitante, il n'y a pas eu de différence de croissance entre les 2 espèces. En condition de sécheresse édaphique, les 2 espèces ont adopté une stratégie se traduisant par une régulation stomatique assez précoce. Le chêne rouge a cependant été rapidement affecté, mais faiblement, tandis que le chêne des marais l'a été plus tardivement, mais fortement (croissance plus faible et surface foliaire plus réduite). Les potentiels hydriques atteints en phase de sécheresse ont été, pour les 2 espèces, de l'ordre de $-1,5$ et de $-2,2$ $\mathrm{MPa}$. Ces résultats sont conformes, pour le chêne rouge, aux données de la littérature et situent les 2 espèces dans une position relativement intermédiaire entre les espèces pratiquant une stratégie d'évitement et celles que l'on peut qualitier de tolérantes. Ces résultats ont ainsi confirmé la connaissance intuitive que l'on avait de l'écologie du chêne des marais par rapport au chêne rouge, et indiquent de ce fait certaines limites à son utilisation forestière.
\end{abstract}

Quercus rubra / Quercus palustris / résistance à la sécheresse / surface foliaire / potentiel hydrique / croissance 


\begin{abstract}
Summary - Seasonal changes in water potential and the growth of young Quercus rubra and Quercus palustris plants during soil drought. In order to compare the resistance of northern red oak (Quercus rubra $L$ ) and pin oak (Quercus palustris Moench) to soil drought, a green-house experiment has been carried out with seedlings grown in containers, where watering was stopped after the first or second growth flush. We measured leaf area, followed height and diameter growth, water potential (seasonal variation), and final biomass. With an optimal watering, both pin oak and northern red oak exhibited a similar height growth. During soil drought, both species exhibited a rather early stomatal regulation but pin oak presented a more severe reduction in height growth and leaf area. During drought, both species displayed a similar range of water potential of about -1.5 to $-2.2 \mathrm{MPa}$. These results are in agreement with the published data for red oak. Both species behaved as mesophilous species and exhibited a strategy intermediate between genuine avoidance and tolerance during a drought. These resuits corroborate our intuitive knowledge of pin oak ecology and point out the limits of its use in forestry.
\end{abstract}

\title{
Quercus rubra / Quercus palustris / drought sensitivity / growth / leaf area / water potential
}

\section{INTRODUCTION}

Introduit de longue date en France (Lanier et al, 1980), le chêne rouge d'Amérique (Quercus rubra $L=Q$ borealis Mich $x$ ) y fait preuve d'une adaptation et d'une croissance remarquable, particulièrement dans le Sud-Ouest (Pilard-Landeau et al, 1986). Du fait de son intérêt forestier, il a fait l'objet d'un assez grand nombre d'études à caractère écophysiologique, tant en France qu'aux ÉtatsUnis (Abrams, 1990 ; Timbal, 1990 ; Cochard et Tyree, 1990 ; Weber et Gates, 1990 ; Colin-Belgrand et al, 1991 ; Kleiner et al, 1991, 1992 ; Timbal et Dreyer, 1994). Le chêne des marais (Quercus palustris Moench) est une autre espèce nord-américaine qui est surtout utilisée, aux ÉtatsUnis comme en France, pour l'ornementation et les plantations d'alignement (Fowells, 1965). Cependant, les quelques essais forestiers existant montrent que, du moins dans le Sud-Ouest et dans certaines conditions de sol, sa croissance est semblable à celle du chêne rouge. De plus, contrairement au chêne rouge, il ne présente pas certains défauts de forme (fourchaison) et de sensibilité à l'encre ou aux sols hydromorphes. Espèce surtout ornementale, le chêne des marais a peu été étudié sur le plant écophysiologique en général et sur celui des relations hydriques en particulier
(Sullivan et Levitt, 1959 ; Borchert, 1975 ; Mac Carthy et Dawson, 1991 ; Colin-Belgrand et al, 1991).

Sullivan et Levitt (1959) avaient déjà comparé les stratégies de réponse à la sécheresse de jeunes plants de chêne rouge et du chêne des marais, mais uniquement sur le plan de l'acquisition d'une résistance à la sécheresse par un préconditionnement consistant à les soumettre à une alimentation en eau préalable restreinte. De plus, les termes de "tolérance» et de "résistance» qu'ils utilisent ne se rapportent pas au fonctionnement stomatique et à la régulation de la transpiration foliaire qu'il permet. C'est pourquoi, il nous a paru intéressant d'étudier de façon comparée la résistance à un dessèchement édaphique de ces 2 espèces de chênes américains, en étudiant l'évolution saisonnière du potentiel hydrique au cours de différentes modalités de dessèchement du sol.

\section{MATÉRIELS ET MÉTHODES}

\section{Matériel végétal}

Les plants de chêne rouge et de chêne des marais utilisés dans l'expérimentation sont des plants dans leur première saison de végétation qui 
ont été obtenus à partir de glands récoltés sur des peuplements locaux (et donc artificiels) :

- chênes rouges : parc du domaine INRA de l'Hermitage à Pierroton (Gironde) ;

- chênes des marais : dans une petite parcelle de la forêt communale de Pujo (Hautes-Pyrénées).

Les glands de chêne rouge ont été triés selon leur grosseur de manière à former un lot homogène quant à leur poids et leur taille. Cette opération n'a pas été nécessaire avec les glands de chêne des marais qui sont beaucoup plus petits et de taille beaucoup plus homogène.

\section{Conteneurs et substrats de culture}

Les glands ont été installés à la surface de conteneurs de grande capacité (environ $70 \mathrm{l}$ ) à raison de 6 par conteneur (5 en périphérie et 1 central). Le substrat de culture était constitué d'un mélange de $90 \%$ de limons (horizon $A_{1}$ d'un sol limoneux acide prélevé en forêt du Mas-d'Agenais dans le département de Lot-et-garonne) et de $10 \%$ de sable blanc minéral landais ; le tout repose sur un lit de graviers siliceux d'environ $10 \mathrm{~cm}$ d'épaisseur. Ce limon acide a une texture caractérisée par environ $15 \%$ d'argile, $30 \%$ de limons, $30 \%$ de sables très fins, $17 \%$ de sables fins et de $8 \%$ de sables grossiers. Son $\mathrm{pH}$ (à l'eau) est de 4,2.

\section{Protocole expérimental}

Pour chacune des 2 espèces, nous avons appliqué 3 régimes hydriques différents :

- Témoin (T) : alimentation hydrique optimale ; les conteneurs étant en permanence alimentés par la base ;

- Sécheresse $1\left(S_{1}\right)$ : arrêt des apports d'eau après le 1er cycle de croissance ;

- Sécheresse $2\left(S_{2}\right)$ : arrêt des apports d'eau après le $2^{\ominus}$ cycle de croissance.

En raison d'une hétérogénéité importante de la croissance des semis, nous nous sommes basés sur le moment où la majorité des semis a atteint le stade phénologique choisi, pour déterminer le moment d'application des sécheresses 1 et 2 (tableau I).
Tableau I. Date d'arrêt d'irrigation des différentes modalités de sécheresse.

$\begin{array}{lcc}\text { Espèces } & S_{1}\left(1^{e r} \text { cycle }\right) & S_{2}\left(2^{e} \text { cycle }\right) \\ & & - \\ \text { Quercus rubra } & 26 / 6 / 89 & 1 / 8 / 89 \\ \text { Quercus palustris } & 26 / 6 / 89 & 18 / 7 / 89\end{array}$

La modalité "témoin» était représentée par 9 conteneurs qui correspondaient donc à autant de répétitions. Les modalités $S_{1}$ et $S_{2}$ étaient représentées par 6 conteneurs, soit 36 plants.

L'expérimentation s'est déroulée dans une serre de type "tunnel» pendant une saison de végétation. II s'agit d'une serre plastique refroidie par un système de cooling. L'éclairement y est environ de $80 \%$ de la pleine lumière. Au cours de la saison de végétation, à l'intérieur de cette serre, la température a varié de 15 à $30^{\circ} \mathrm{C}$ et l'humidité relative de 30 à $70 \%$.

Les conteneurs y ont été déposés sur 2 rangées parallèles et contiguës (une rangée de chênes rouges et une rangée de chênes des marais) ; chacune regroupant, de manière aléatoire, les répétitions des différents traitements (blocs éclatés).

\section{Mesures}

\section{Humidité du substrat}

Dans les modalités $S_{1}$ et $S_{2}$, le dessèchement du sol a été suivi par la mesure de l'humidité à 15 et à $30 \mathrm{~cm}$ de profondeur au moyen de sondes à plâtre (Nardeux) reliées à un pédohygromètre portable (Eijkelkamp). Ce dernier ne donnant que des résultats qualitatifs, il a été nécessaire de l'étalonner de manière à pouvoir convertir les valeurs affichées en une expression de l'humidité pondérale du sol. L'étalonnage a consisté, après mesures au pédohydromètre, à prélever dans le conteneur, au même niveau ( -15 et $-30 \mathrm{~cm}$ ), une carotte de sol pour en définir l'humidité pondérale par pesée avant et après passage à l'étuve à $105^{\circ} \mathrm{C}$ pendant $24 \mathrm{~h}$. 


\section{Croissance des plants et biomasse}

Pour chacune des 2 espèces comparées, la hauteur totale et le diamètre au collet ont été mesurés, d'abord au moment de l'application des sécheresses $S_{1}$ et $S_{2}$, c'est-à-dire respectivement après les $1^{\text {er }}$ et $2^{e}$ cycles de croissance et ensuite en fin d'expérience, après la chute des feuilles à l'automne.

La biomasse anhydre des tiges (sans les feuilles) et des racines a été également mesurée en fin de saison de végétation après passage à l'étuve à $60^{\circ}$ pendant $48 \mathrm{~h}$. La biomasse des feuilles n'a pu être mesurée en raison de leur chute très échelonnée dans le temps.

\section{Surfaces foliaires}

En fin de saison de végétation, avant le début de la chute des feuilles, nous avons compté le nombre de feuilles par plant et mesuré leurs dimensions principales (longueur et largeur maximum du limbe) afin de déterminer les surfaces foliaires par plant, par conteneur, par modalité et par espèce. Pour chaque feuille, la surface foliaire a été estimée à l'aide d'un «tarif» dont les entrées sont la longueur et la largeur maximum du limbe ; tarif différent pour chaque cycle de croissance. Pour le chêne rouge, nous avons utilisé un tarif préexistant (Jung-Muller, 1987). Pour le chêne des marais, nous avons établi un tarif en déterminant, sur un échantillon représentatif, le coefficient de la régression entre la surface exacte des limbes, mesurée à l'aide d'une table à numériser, et le produit longueur $x$ largeur maximale du limbe (voir annexe).

\section{État hydrique des plants}

Au cours du dessèchement progressif du sol dans les modalités $S_{1}$ et $S_{2}$, le potentiel hydrique des feuilles (potentiel de base et potentiel minimum) a été mesuré, chaque semaine, à l'aide d'une chambre à pression, sur une feuille prélevée sur un des 6 plants de chacun des 6 (ou 9) conteneurs de chacune des 3 modalités de chaque espèce. Le plant du conteneur sur lequel la feuille était prélevée (feuille du dernier verticille mis en place) changeait chaque semaine. Cela s'est traduit par une périodicité de prélèvement de feuille par plant de 6 sem.

Les mesures de potentiel hydrique sur feuile ont débuté 1 sem après l'arrêt de l'arrosage (modalité $S_{1}$ ) sur le chêne rouge et le 8/8/90, c'est-à-dire $1 \mathrm{sem}$ après la mise en place de la modalité $S_{2}$, sur le chêne des marais. Ce déla a été dû au fait que les feuilles du $1^{\text {er }}$ cycle se sont révélées trop petites (pétiole trop court, voire inexistant, et limbe trop étroit), pour des mesures à la chambre à pression.

Lors de chaque série de mesure, les 3 feuilles, prélevées sur les différents traitements, ont été stockées en sachet de polyéthylène (1 feuille par sachet) et en glacière, selon la procédure mise au point par El Hadj Moussa (1986).

\section{RÉSULTATS}

\section{Évolution de l'humidité du sol}

La figure $1(a, b, c, d)$ présente l'évolution saisonnière des valeurs de l'humidité pondérale du sol à -15 et $-30 \mathrm{~cm}$, exprimées en fraction de l'humidité maximale.

D'une manière générale, quelles que soient l'espèce et la modalité, les niveaux de sécheresse atteints ne sont devenus importants qu'à partir de la $6^{\mathrm{e}}$ semaine. Par la suite, l'assèchement s'est poursuivi rapidement.

Les formes des courbes d'assèchement ont présenté une évolution similaire : d'abord très lente, puis rapide, puis à nouveau lente. La position relative des courbes correspondant aux modalités $S_{1}$ et $S_{2}$ est généralement la même: dessèchement plus rapide dans la modalité $S_{2}$, à mettre sans doute en relation avec une transpiration plus élevée des plants, elle-même liée à une surface foliaire plus importante. Cependant, avec Quercus palustris et à $-15 \mathrm{~cm}$, le dessèchement a été plus rapide dans la modalité $S_{1}$ que dans la modalité $S_{2}$. Dans ce dernier cas, on peut penser qu'avec une surface foliaire développée plus faible (cf infra) l'évaporation directe au niveau de la surface des conteneurs a été plus importante. 

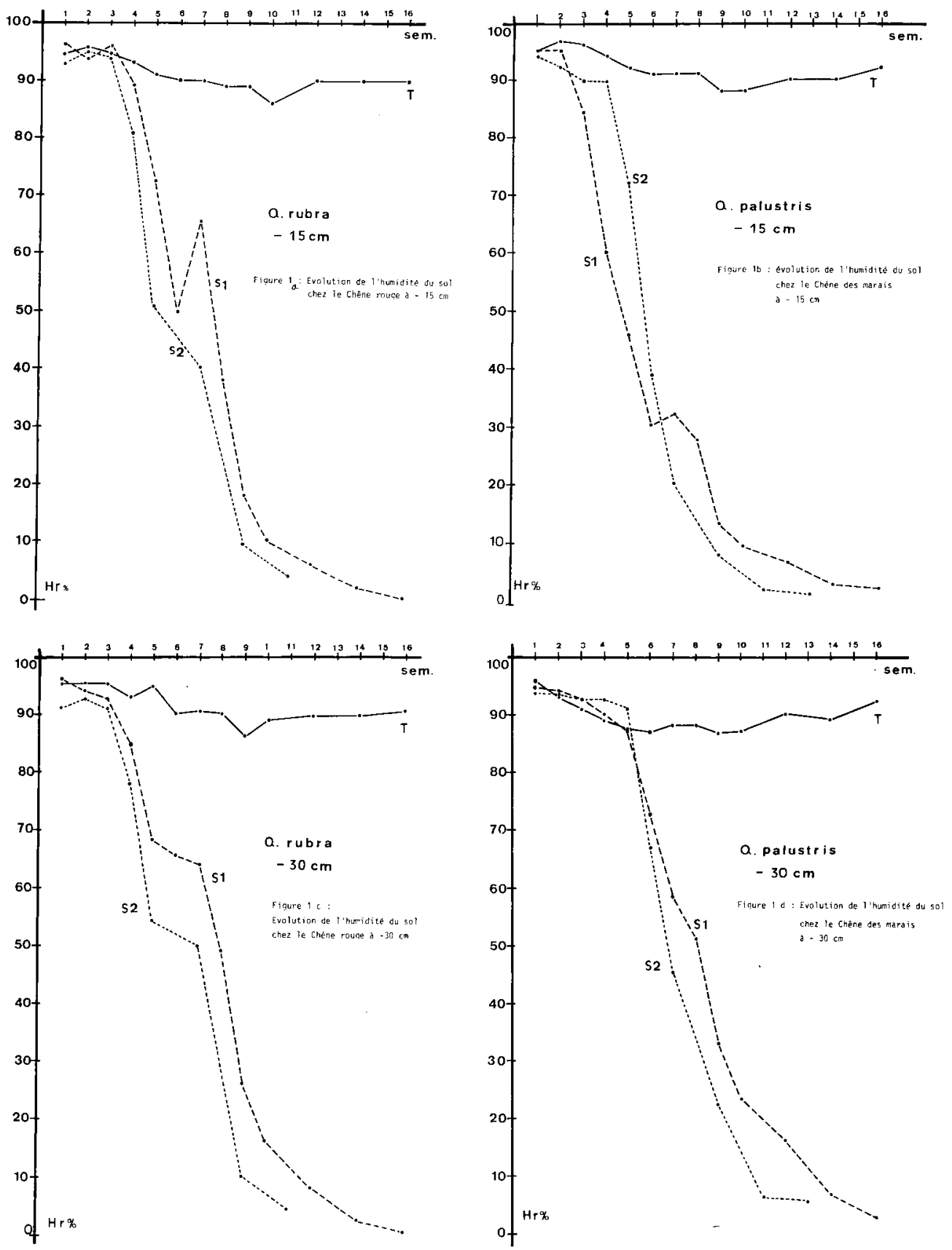

Fig 1. a. Évolution de l'humidité du sol chez le chêne rouge à $-15 \mathrm{~cm} ; \mathbf{b}$. évolution de l'humidité du sol chez le chêne des marais à $-15 \mathrm{~cm}$; c. évolution de l'humidité du sol chez le chêne rouge à $-30 \mathrm{~cm}$; d. évolution de l'humidité du sol chez le chêne des marais à $-30 \mathrm{~cm}$. 
Le tableau II indique la durée nécessaire pour obtenir une baisse de $50 \%$ de l'humidité du sol aux différentes profondeurs.

\section{Surfaces foliaires}

\section{Nombre de feuilles (fig 2)}

En alimentation en eau non limitante (témoins), les semis de chêne rouge ont un

Tableau II. Nombre de semaines nécessaires pour atteindre un dessèchement du substrat correspondant à une humidité pondérale de $50 \%$ en fonction du traitement et de la profondeur.

Espèces

\begin{tabular}{|c|c|c|c|}
\hline & & $15 \mathrm{~cm}$ & $-30 \mathrm{~cm}$ \\
\hline Q rubra & $\begin{array}{l}S_{1} \\
S_{2}\end{array}$ & $\begin{array}{l}6 \\
5\end{array}$ & $\begin{array}{l}8 \\
7\end{array}$ \\
\hline Q palustris & $\begin{array}{l}S_{1} \\
S_{2}\end{array}$ & $\begin{array}{l}4,5 \\
5,5\end{array}$ & $\begin{array}{l}8 \\
6,5\end{array}$ \\
\hline
\end{tabular}

nombre de feuilles très supérieur à ceux du chêne des marais. En conditions de sécheresse édaphique, on note, chez les 2 espèces, une diminution du nombre final de feuilles par plant.

\section{Surface individuelle des feuilles (tableau III)}

Les semis de chêne rouge ont présenté des feuilles plus grandes que ceux des chênes des marais, et ce lors de tous les cycles de croissance, et quel qu'ait été le traitement appliqué. Dans tous les cas aussi, la surface des feuilles a augmenté lors du passage aux cycles de croissance plus tardifs; en particulier, la surface des feuilles du $3^{e}$ cycle a pratiquement triplé chez Quercus palustris.

\section{Surfaces foliaires totales (fig 3)}

En condition d'alimentation en eau non limitante (témoins), les semis de chêne rouge ont présenté une surface foliaire totale par plant très supérieure à ceux du chêne des marais (davantage de feuilles et des feuilles

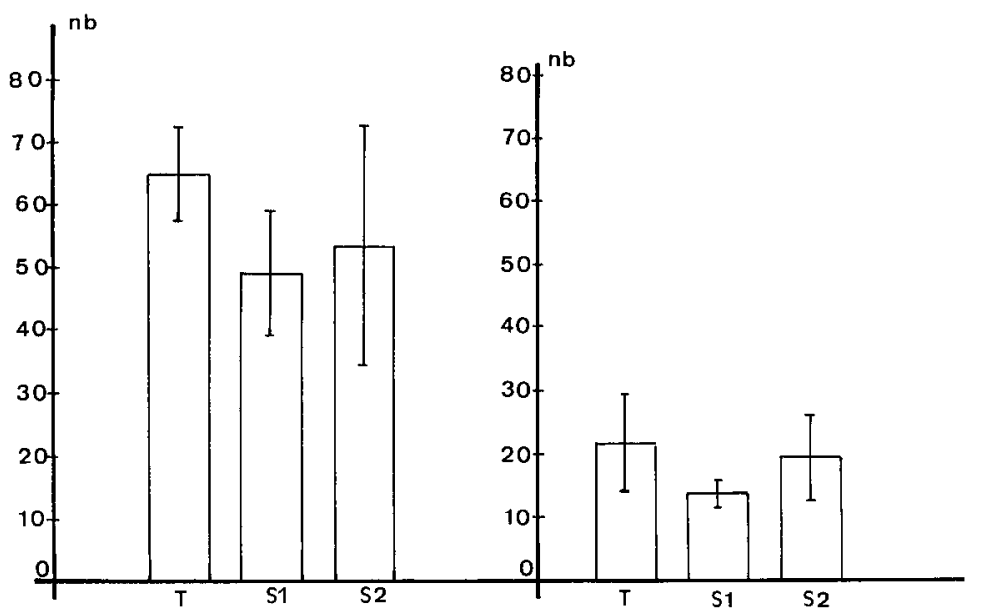

A: Q.rubra
$B: Q$. palustris
Fig 2. Nombre moyen de feuilles par modalité. $T=$ témoin, $\mathrm{S}_{1}=$ sécheresse au $1^{\text {er }}$ cycle, $\mathrm{S}_{2}=$ sécheresse au $2^{e}$ cycle. 
Tableau III. Surface moyenne des feuilles $\left(\mathrm{cm}^{2}\right)$ des 3 premiers cycles de croissance, en fonction des traitements.

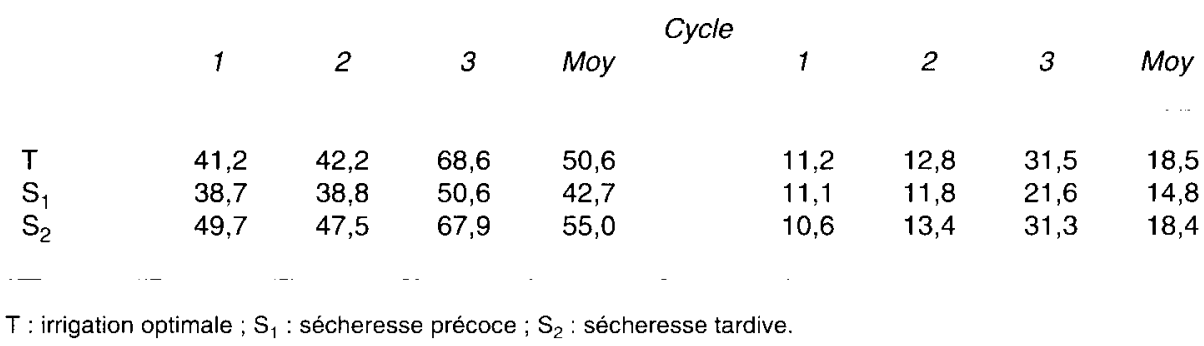

plus grandes). En condition de sécheresse édaphique, cette surface foliaire totale (St) a été significativement réduite. L'intensité de cette réduction différait selon la modalité de sécheresse et l'espèce : elle a été plus forte chez Quercus palustris que chez Quercus rubra et plus marquée en réponse au traitement $S_{1}$. Cependant, chez Quercus rubra et pour $S_{2}$, la différence par rapport au témoin n'est pas significative.

Donc, non seulement le chêne des marais présentait une surface foliaire plus faible que celle du chêne rouge en condi- tions optimales, mais encore une sécheresse édaphique a provoqué une réduction de cette surface foliaire plus marquée que sur le chêne rouge.

\section{Potentiel hydrique des plants (fig 4a,b)}

En condition hydrique non limitante (témoin), le potentiel hydrique de base a varié au cours de la saison de végétation de $-0,05$ à $-0,28 \mathrm{MPa}$ (la dernière semaine) pour le chêne rouge et de $-0,21$ à $-0,60 \mathrm{MPa}$ pour

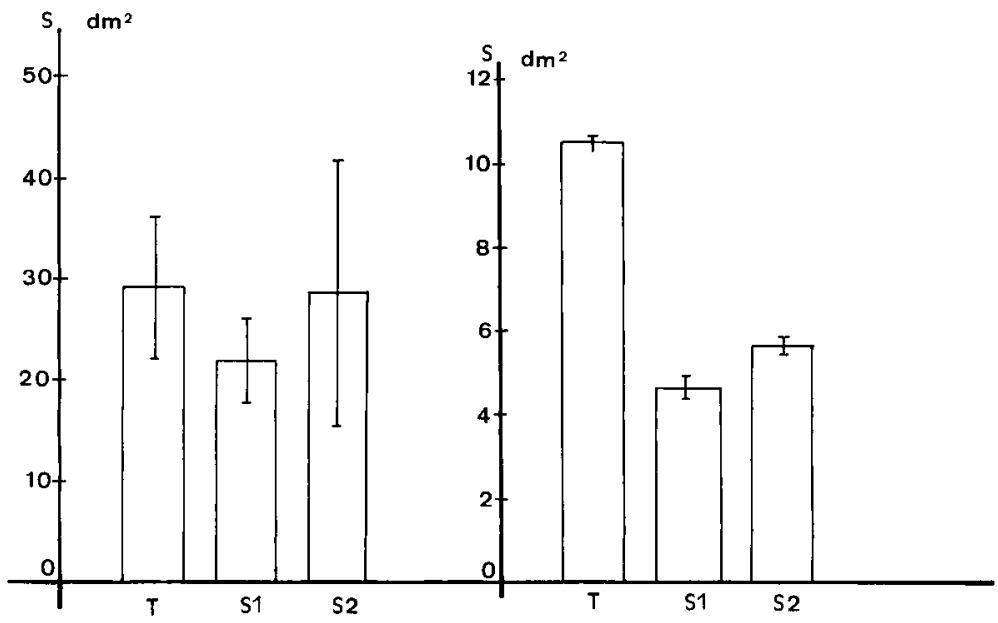

A: a. rubra
B : a.palustris
Fig 3. Surface foliaire totale selon les traitements. $\mathrm{T}=$ témoin, $S_{1}=$ sécheresse au 1er cycle, $S_{2}=$ sécheresse au $2^{e}$ cycle. 
le chêne des marais. Pendant la même période, le potentiel minimum, atteint au milieu de la journée quand le soleil est à son zénith, a varié de $-0,6$ à $-1,23 \mathrm{MPa}$ pour le chêne rouge, et de $-0,92\left(6^{\mathrm{e}}\right.$ semaine) à -1,8 $\mathrm{MPa}$ pour le chêne des marais.

En condition d'assèchement du sol, ce n'est qu'au bout de 3 sem que les effets de l'arrêt des arrosages sur le potentiel hydrique de base se font sentir, et cela quelles qu'aient été l'espèce et la modalité (fig 4). Pour Quercus rubra, les valeurs du potentiel hydrique de base ont diminué régulièrement en fonction du degré de dessèchement du sol. Les courbes relatives aux modalités $S_{1}$ et $S_{2}$ sont similaires, mais évidemment décalées l'une par rapport à l'autre. Pour une même durée de dessè- chement (11 sem), le potentiel de base est descendu à -0,73 MPa (valeur calculée par interpolation) pour la modalité $S_{1}$ et à $-1,30$ MPa pour $S_{2}$. L'humidité relative du sol est alors de $10 \%$ à $-15 \mathrm{~cm}$ (et de $15 \%$ à -30 $\mathrm{cm}$ ) dans la modalité $S_{1}$ et de $5 \%$ (à -15 et $-30 \mathrm{~cm}$ ) dans la modalité $S_{2}$. Ces différences sont bien entendu à mettre en rapport avec les différences de surface foliaire développées dans ces 2 modalités, et avec les différences d'évapotranspiration correspondantes.

Pour Quercus palustris, l'évolution du potentiel hydrique de base en fonction de l'assèchement du substrat a été légèrement différente: les différences entre modalités se sont fortement accentuées au bout de la $9^{e}$ sem. Ainsi, à la $11^{e}$ sem, le potentiel de base est en moyenne de $-0,5 \mathrm{MPa}$
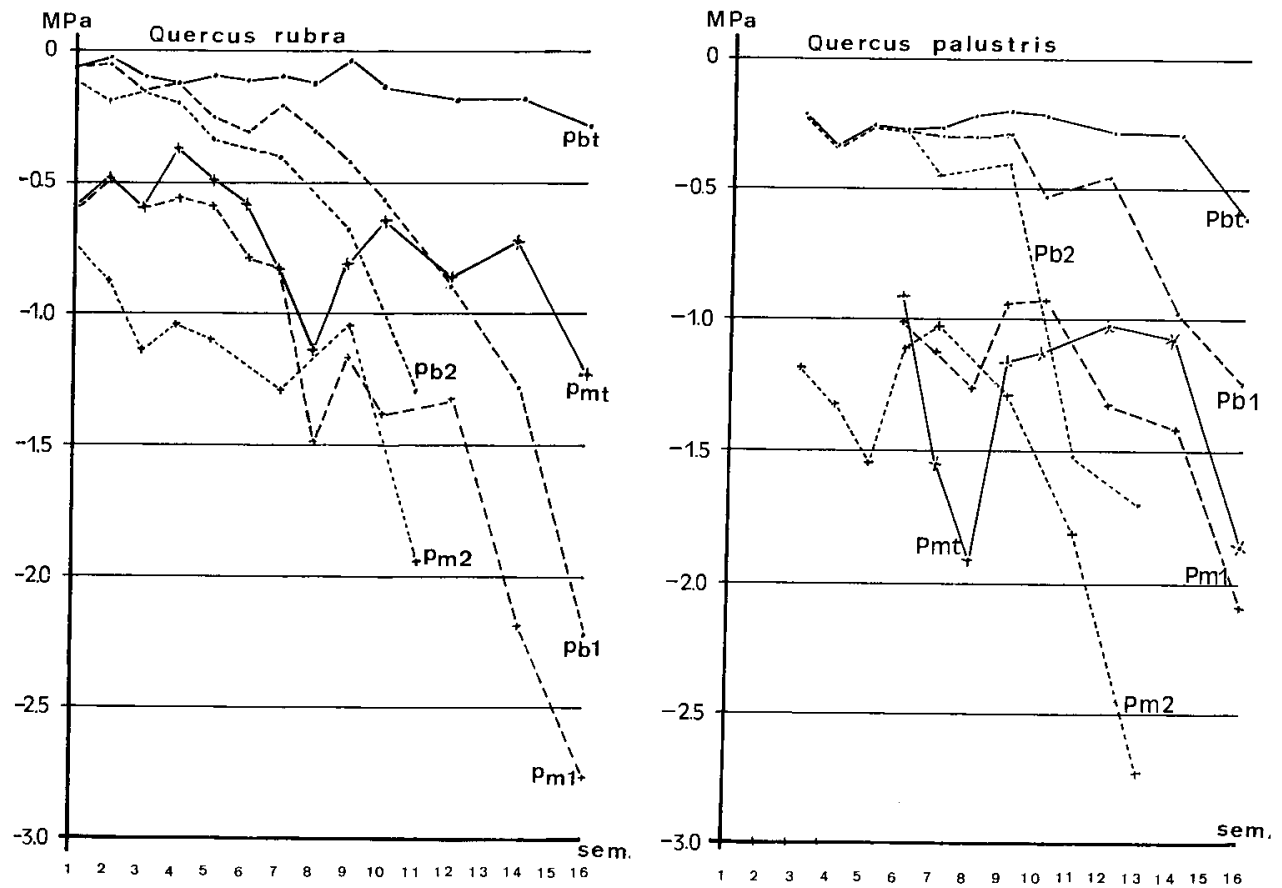

Fig 4. a. Évolution du potentiel hydrique pour le chêne rouge selon les modalités. $\mathrm{Pb}$ : potentiel de base ; $\mathrm{Pm}$ : potentiel minimum ; $\mathrm{t}$ témoin ; 1 : sécheresse 1 er cycle $; 2$ : sécheresse $2^{\ominus} \mathrm{cycle}$. b. évolution du potentiel hydrique pour le chêne des marais selon les modalités. $\mathrm{Pb}$ : potentiel de base ; Pm : potentiel minimum ; $T$ : témoin ; 1 : sécheresse $1^{\text {er }}$ cycle ; $2:$ sécheresse $2^{e}$ cycle. 
(valeur par interpolation) dans la modalité $\mathrm{S}_{1}$ et de $-1,5 \mathrm{MPa}$ dans la modalité $\mathrm{S}_{2}$. Les valeurs atteintes les plus faibles sont de $-2,2 \mathrm{MPa}$ pour Quercus rubra (modalité $\mathrm{S}_{1}$ ) et de $-1,7 \mathrm{MPa}$ pour Quercus palustris (modalité $\mathrm{S}_{2}$ ).

De telles valeurs de potentiel de base et de potentiel minimum en condition de sécheresse édaphique sont tout à fait comparables à celles déjà publiées pour le chêne rouge (Abrams, 1990 ; El Hadj Moussa, 1986 ; Weber et Gates, 1990). Bien que faibles, ces valeurs mesurées ne correspondent sans doute pas encore au potentiel de base critique. En effet, dans une expérimentation antérieure (Timbal et Gelpe, 1988 , non publié), des potentiels de base de $-3,0$ à 3,3 MPa avaient été mesurés sur des plants de chêne rouge d'un an soumis, pendant 8 à 9 sem, à un assèchement total ; ces plants avaient néanmoins retrouvé un potentiel de base de $-0,3 \mathrm{MPa}$ après réhumectation. À titre de comparaison, nous avons mesuré le potentiel hydrique de base sur des feuilles de rejets de chêne rouge poussant sur le sol sableaux du domaine INRA de l'Hermitage à Cestas (Gironde), à la fin des étés 1989 et 1990 , années toutes 2 caractérisées par une très forte sécheresse estivale. Les valeurs trouvées étaient de $-2,2 \mathrm{MPa}$ en 1989 et de -1,5 MPa en 1990.

Dans les modalités d'assèchement $S_{1}$ et $S_{2}$, les différences de potentiel de base observées en condition d'alimentation hydrique non limitante entre Quercus rubra et Quercus palustris se sont atténuées mais n'ont pas disparu. Le chêne des marais atteint plus rapidement que le chêne rouge un potentiel hydrique donné. Ainsi, un potentiel de base de -1,0 MPa est atteint par le chêne des marais au bout de 7 sem de dessèchement dans la modalité $S_{2}\left(8\right.$ dans $S_{1}$ ), et par le chêne rouge, toujours pour $S_{2}$, au bout de 14 sem (11 dans S1).

\section{Croissance}

\section{Croissance en hauteur (fig 5)}

En condition d'alimentation en eau optimale, il n'y a pas de différence significative sur les hauteurs totales entre les 2 espèces de chêne $(30 \mathrm{~cm})$. Une sécheresse édaphique a entraîné une différence spécifique de croissance, faible et non significative chez le chêne rouge, plus importante et significative chez le chêne des marais. Cette réduction, qui a touché les cycles de croissance 2, 3 et 4 est de l'ordre de 30 à $35 \%$.

\section{Croissance en circonférence au collet}

Les résultats sont les mêmes que pour la croissance en hauteur. En condition d'alimentation en eau non limitante il n'y a pas de différence significative entre les espèces. En condition de sécheresse édaphique, il n'y pas de différence significative avec le témoin chez Quercus rubra tandis que chez Quercus palustris il existe une difference significative de diamètre au collet dans la seule modalité $S_{1}$.

\section{Croissance en biomasse (tableau IV)}

La biomasse racinaire des semis de Quercus palustris était significativement plus élevée que celle de Quercus rubra en l'absence de contrainte. En condition de sécheresse édaphique (modalités $S_{1}$ et $S_{2}$ ), il n'y avait pas de différences entre espèces pour les biomasses des tiges et des racines (et donc aussi pour les biomasses totales), mais des différences significatives entre traitements pour une espèce donnée. Ainsi, pour le chêne rouge, les 3 traitements donnent des résultats significativement différents pour la biomasse totale. Pour le chêne des marais, les racines, les tiges, ou la somme tiges + racines ont été significati- 


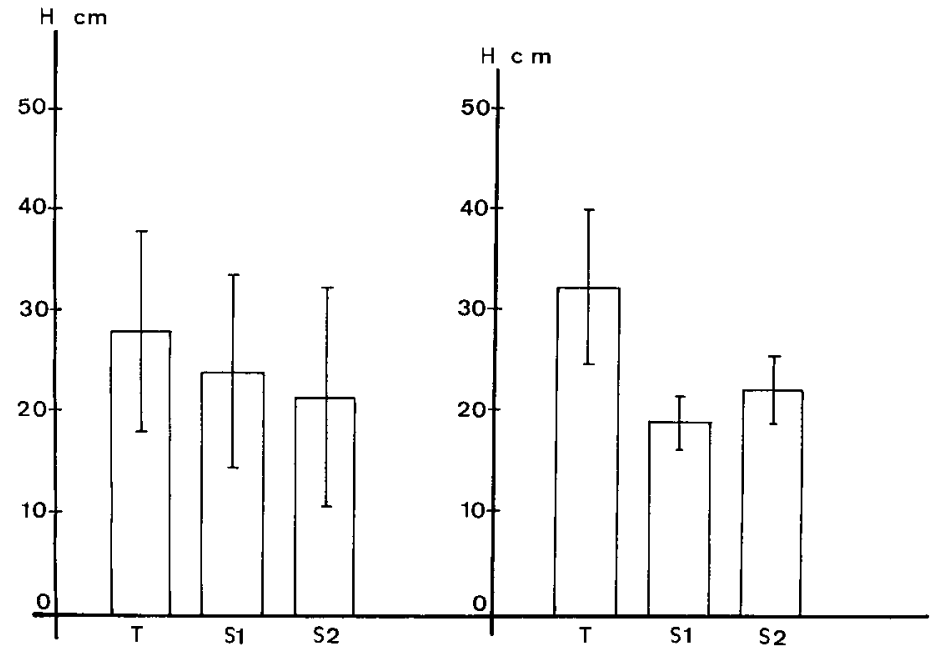

A: Q. rubra

B: Q. palustris
Fig 5. Croissance en hauteur selon les modalités. $\mathrm{T}=$ témoin, $S_{1}=$ sécheresse au 1 er cycle, $\mathrm{S}_{2}=$ sécheresse au $2^{\mathrm{e}}$ cycle.

Tableau IV. Biomasses moyennes par traitement (en g).

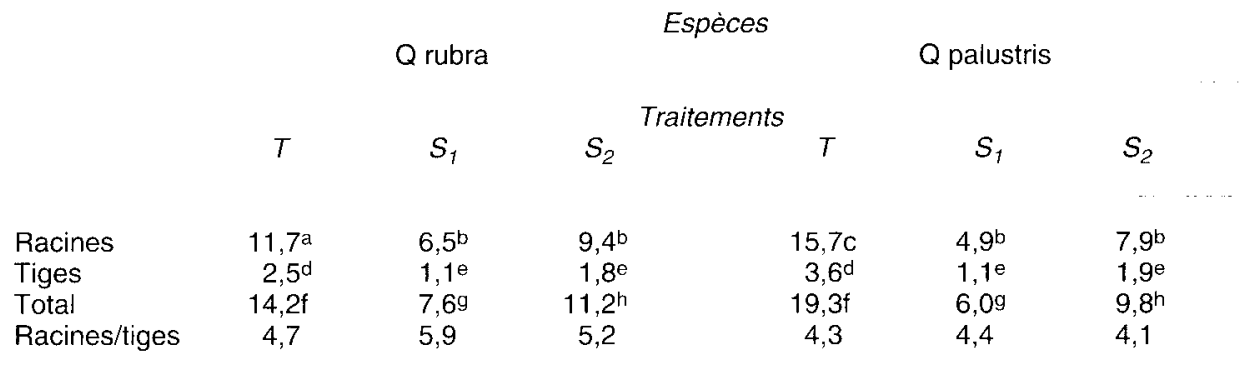

2 valeurs suivies de la même lettre ne sont pas significativement différentes au seuil de $5 \%$.

vement affectés par les traitements de sécheresse mis en œuvre.

\section{DISCUSSION ET CONCLUSIONS}

En condition d'alimentation en eau optimale, des similitudes et des différences de comportement entre semis de Quercus rubra et Quercus palustris ont été mis en évidence. II n'y a pas eu de différence de croissance en hauteur entre les 2 espèces, mais il y en a eu une pour les biomasses racinaires (et totales) au profit du chêne des marais. Cela est d'autant plus remarquable que le chêne des marais a des glands nettement plus petits que le chêne rouge (et donc d'autant moins de réserves) et qu'il a développé une surface foliaire plus réduite.

Les semis de Quercus palustris ont ainsi fait preuve d'une forte efficience de croissance comparés à ceux de Quercus rubra. La biomasse racinaire a été concentrée 
dans un pivot important plus que dans de nombreuses fines racines. On admet généralement qu'une telle allocation préférentielle de biomasse vers les racines constitue un bon critère d'adaptation à la sécheresse (Kolb et al, 1990).

Malgré une surface foliaire plus petite, Quercus palustris a asséché plus vite son substrat que Quercus rubra. Cela voudrait dire qu'il transpire davantage et qu'il a une conductance stomatique plus élevée en conditions de faible sécheresse.

La sécheresse édaphique a conduit a une amplification de ces différences de comportement ; le chêne des marais montrant, dans l'ensemble, une sensibilité plus grande, ce que l'analyse de la croissance par cycle permet de mieux comprendre. Les semis de chêne rouge ont réagi vite mais peu à la sécheresse édaphique alors que le chêne des marais a réagi plus tardivement mais plus fortement. De plus, Quercus rubra et Quercus palustris ont présenté des rythmes de croissance très différents. Ces différences sont sans doute à mettre en relation avec la quantité des réserves contenues dans les glands et les surfaces foliaires développées durant les différents cycles de croissance.

Au terme de cette étude, des différences assez nettes de comportement vis-à-vis d'une sécheresse édaphique apparaissant entre le chêne rouge et le chêne des marais.

Sur le plan de la morphologie foliaire, pour le chêne des marais, la réaction à la sécheresse se traduit par une réduction très nette de la surface foliaire (moins de feuilles et des feuilles plus petites), ce qui constitue une manière de réduire la transpiration et, partant, la photosynthèse. De plus, le chêne des marais semble réguler plus précocement que le chêne rouge son potentiel hydrique foliaire, ce qui constitue également un mécanisme de résistance à la sécheresse.
Du fait de la forte réserve hydrique du substrat et de la faible transpiration des plants de Quercus palustris (faible surface foliaire et régulation stomatique précoce), il n'a pas été possible d'atteindre le potentiel de base critique. On peut cependant penser qu'il doit être inférieur à $-1,9 \mathrm{MPa}$, chiffre d'ailleurs conforme aux données citées dans la littérature (Abrams, 1990).

Malgré sa plus grande sensibilité à la sécheresse, le chêne des marais a finalement présenté un comportement hydrique assez semblable à celui du chêne rouge. Comme lui, il adopte, vis-à-vis de l'eau, une stratégie intermédiaire entre celle qualifiée d' "évitement" (comme les bouleaux) consistant en une régulation stomatique très précoce, et celle qualifiée de "résistance" (comme les cèdres et beaucoup d'espèces méditerranéennes). Le résultats obtenus montrent d'ailleurs que, le long de ce continuum, le chêne des marais a une stratégie qui est plus proche de l'«évitement»que le chêne rouge. Comme lui, on peut le ranger dans la catégorie des espèces mésophiles, alors que son nom aurait laissé présager un comportement plus franchement mésohygrophile, voire hygrophile.

Cependant, la mesure du potentiel hydrique (potentiel de base et potentiel minimum) ne suffit pas à caractériser complètement le comportement d'une espèce face à un stress hydrique. Il faudrait aussi suivre parallèlement les échanges gazeux et donc l'évolution de la conductance stomatique, ce qu'il ne nous a pas été possible de faire au cours de cette expérimentation.

Sur le plan pratique, nous avons vu que le chêne des marais montre, pour une surface foliaire moindre, une croissance en hauteur et en diamètre équivalente à celle du chêne rouge, ce qui constitue un de ses intérêts sylvicoles et donne une indication sur ses limites d'utilisation. Une indication seulement, car il serait nécessaire de comparer également ces 2 espèces de chêne sur d'autres plants et, en particulier, sur celui 
de la résistance à l'anoxie. Dans cette voie, peu de résultats ont été obtenus : Black (1987), Dreyer et al (1991). En particulier, ces derniers n'ont pas trouvé de différences entre les jeunes semis de ces 2 espèces en ce qui concerne l'influence de l'anoxie sur les capacités photosynthétiques.

\section{ANNEXE}

\section{Détermination des surfaces foliaires}

\section{Feuilles de Quercus palustris}

La détermination des surfaces foliaires unitaires a été effectuée, pour chaque cycle de croissance, à partir des mesures de la longueur et de la largeur maximum du limbe. Le produit $\left(^{*}\right)$ de la longueur (L) par la largeur (I), rapporté à la mesure de la surface réelle au moyen d'une table à numériser, a été corrigé dans un premier temps par les coefficients $A^{\prime}{ }_{0}$ et $A^{\prime}{ }_{1}$ déterminés par la régression simple:

$$
\text { Surface approchée }=\left(L^{*} 1\right)^{*} A_{1}^{\prime}+A_{0}^{\prime}
$$

Dans un deuxième temps, cette surface approchée est rapportée à la surface réelle mesurée à la table à numériser, ce qui permet la détermination des coefficients finaux $\mathrm{A} 0$ et $\mathrm{A} 1$ de la régression. L'équation finale retenue est donc:

$$
\text { Surface réelle }=\left(L^{\star} 1\right){ }^{\star} A_{1} \text { et } A_{0}
$$

Ces coefficients ont été déterminés pour chacun des 4 premiers cycles de croissance.

$\begin{array}{ccc}\text { Cycle } & \boldsymbol{A}_{0} & \boldsymbol{A}_{1} \\ & & \\ 1 & 0,0000 & 1,0000 \\ 2 & 0,9670 & 1,0002 \\ 3 & 0,0008 & 1,1333 \\ 4 & 0,0022 & 1,0000\end{array}$

\section{Feuilles de Quercus rubra}

Pour le chêne rouge, une équation similaire a été déterminée lors d'une précédente étude (Jung-Muller, 1987).

Surface $=\left(L^{1,12185 *} A_{1}\right) *\left(10,70259 * A_{2}\right)+B$

avec: $A_{1}=0,54838$ et $A_{2}=0,978 ; B=4,917$ pour le $1^{\circ}$ cycle, $=-7,569$ pour le $2^{\circ}$ cycle, $=-2,199$ pour le $3^{\circ}$ cycle.

\section{RÉFÉRENCES}

Abrams MD (1990) Adaptation and response to drought in Quercus sp of North America. Tree Physiol 7, 227. 238

Black RA (1984) Water relations of Quercus palustris: field measurements of an experimentally flooded stands. Oecologia 64, 14-20

Borchert R (1975) Endogenous shoot growth rhythms and indeterminate shoot growth in oak. Physiol Plant 35, 152-157

Cochard H, Tyree MT (1990) Xylem dysfunction in Quercus: vessel sizes, tyloses, cavitation and seasonal changes in embolism. Tree Physio/ 6, 393-407

Colin-Belgrand M, Dreyer E, Biron P (1991) Sensitivity of seedlings from different oak species to waterlogging: effects on root growth and mineral nutrition. Ann Sci For 48, 193-204

Dreyer E, Colin-Belgrand M, Biron P (1991) Photosynthesis and shoot water status of seedlings from different oak species submitted to waterlogging. Ann Sci For 48,205-214

El Hadj Moussa F (1986) Contribution à l'étude écophysiologique du comportement hydrique chez Quercus rubra. Mémoire de DEA, université de Pau (IBEAS) et laboratoire INRA de sylviculture et écologie, $45 \mathrm{p}$

Fowells $H$ (1965) Silvics of forest trees of the United States. USDA Agriculture handbook, $271 \mathrm{p}$

Jung-Muller B (1987) Variabilité morphologique et électrophorétique des peuplements introduits de chêne rouge (Quercus rubra L). Mémoire de stage $3^{\circ}$ année ENITEF, laboratoire INRA de génétique et amélioration, $87 p$

Kleiner KW, Abrams MD, Schultz JC (1992) The impact of water and nutrient deficiencies on the growth, gas exchange and water relations of red oak and chestnut oak. Tree Physiol 7, 215-225

Kolb TE, Steiner KC, Mac Cormick LH, Bowersox TW (1990) Growth response of northern red oak and 
Yellow poplar seedlings to light, moisture and nutrients in relation to ecological strategy. For Ecol Manag 38, 65-78

Lanier L, Keller R, Kremer A (1980) Le chêne rouge en France (Quercus rubra) L). Rev For Fr 32, 419-451

Mac Carthy JJ, Dawson JO (1991) Effect of drought and shade on growth and water use of Quercus alba, $Q$ bicolor, $Q$ imbricaria and $Q$ palustris seedlings. Proc 8th Cent Hardwood For Conf Univ Park (PA), USDA For Ser Northeastern Exp Stn Gen Tech Rep NE $148,157-178$

Pilard-Landeau B, Gelpe J, Lemoine B, Timbal J (1986) Le chêne rouge d'Amérique (Quercus rubra $\mathrm{L}=Q$ borealis Michx) dans le Sud-Ouest. Rev For Fr 38(1), $27-40$

Sullivan CY, Levitt J (1959) Drought tolerance and avoidance in 2 species of oak. Physiol Plant 12, 299-305

Timbal J (1990) Le chêne rouge d'Amérique : écologie et facteurs limitants. Rev For Fr 42(2), 165-173

Timbal J, Dreyer E (1994) Consommation en eau et résistance à la sécheresse. In : Le chêne rouge, INRA ed, 85-90

Weber JA, Gates DM (1990) Gas exchange in Quercus rubra (northern red oak) during a drought: analysis of relations among photosynthesis, transpiration and leaf conductance. Tree Physiol 7, 215-225 